\title{
Adaptive Local Power-Law Transformation for Color Image Enhancement
}

\author{
Chun-Ming Tsai* \\ Department of Computer Science, Taipei Municipal University of Education, 100 Taipei, Taiwan
}

Received: 4 Feb. 2013, Revised: 5 Jun. 2013, Accepted: 6 Jun. 2013

Published online: 1 Sep. 2013

\begin{abstract}
Color image enhancement is a very important process that has significantly impacted many portable devices, including PDAs, digital cameras, and mobile camera phones. Due to the limited memory space and computational power of portable devices, reducing the computational complexity of an embedded system is priority concern. Thus, this paper proposes an adaptive local powerlaw transformation method with low computational complexity and high performance. In order to reduce the computational time, a fast method is proposed to compute the local mean from the neighboring pixels to adapt the exponential value in the power-law transformation for enhancing the color images. The experiments reported included various color images; the results show that the performance of the proposed method is better than the compared global and local enhancement methods in terms of both non-over enhancement and natural color clearly revealed. In particular, the proposed enhancement method has low computational cost. Moreover, the proposed algorithm can be run on an embedded environment and processed in a real-time system due to its simplicity and efficiency
\end{abstract}

Keywords: Color Image Enhancement, Adaptive Local Power-Law Transformation, Power-Law Transformation, Gamma Correction, Color Images

\section{Introduction}

Many new consumer electronic products have been introduced into the market: Tablet PCs, smart phones, E-book readers, HDTVs, LCD displays, digital cameras, etc. Achieving good image quality has been highly demanding for consumer electronic products because the image quality is deteriorated by the following factors: bit-resolution is limited, dynamic range is narrow, and the acquisition time is insufficient [1]. Contrast enhancement is used to adjust the image quality for better human visual perception [2], and is a very important preprocessing step for tasks in image processing, video processing, medical image processing, aerial image processing, and computer vision. The conventional gray-level contrast enhancement methods can be extended to enhance the color images by color space transformation.

Current contrast enhancement algorithms based on spatial domain techniques can be divided into global and local methods. Global enhancement methods enhance the image from the information (luminance and saturation) of an entire image. Histogram equalization (HE) and its variations are one of the most commonly used algorithms to perform contrast enhancement due to its simplicity and effectiveness. Kim [3] proposed a bi-histogram equalization (BBHE) to preserve the mean brightness. His $\mathrm{BBHE}$ is based on the mean brightness to divide the original histogram into two sub-histograms. Each sub-histogram is enhanced by using the HE method. Wan et al. [4] proposed a dualistic sub-image histogram equalization (DSIHE). The DSIHE is based on using the median brightness to separate the original histogram into two sub-histograms. Each sub-histogram is also enhanced by using HE method. Menotti et al. [5] proposed two novel Multi-HE (MWCMHE and MMLSEMHE) techniques, which consist of decomposing the input image into several sub-images, and applying the classical HE [2] process to each one. Their method can preserve more the brightness and produce more natural looking images than can the other HE methods. However, their method needs to set the weighting constant of the cost function in advance.

Local enhancement methods enhance the image for each pixel according to the information (luminance, saturation, and Retinex) and that of its neighbor. Chatterji and Murthy [6] proposed an adaptive contrast

\footnotetext{
*Corresponding author e-mail: cmtsai2009@gmail.com
} 
enhancement for color images. However, their method needs to determine two parameters: enhancement function and region size in advance. Meylan and Ssstrunk [7] used a Retinex-based adaptive filter to enhance natural color images, and their results showed that the color image with halo area could be enhanced. However, their method required choosing the appropriate filter size to reduce halos images and to introduce global tone mapping for extremely high dynamic range images. Munteanu and Rosa [8] used a GA algorithm to search the parameters of the Multi-Scale Retinex model; in their model, eleven parameters (seven for Retinex, four for GA) needed to be set. Jobson et al. [9] proposed a multi scale retinex with color restoration (MSRCR) to restore color. The color value of a pixel is computed by taking the ratio of the pixel to the weighted average of the neighboring pixels. This method produces abnormal color shifts and halo effects [10]. Kimmel et al. [11] proposed a retinex-based gamma correction to reduce the halo effects. However, this method does not determine the gamma value automatically. Tao and Asari [12] proposed an adaptive and integrated neighborhood-dependent approach for nonlinear enhancement (AINDANE) for low and non-uniform illumination images. Capra et al. [13] used a bilateral filter to develop the dynamic range optimization (DRO) for shadow and highlight contrast correction.

Recently, $\mathrm{Xu}$ et al [14] proposed an object-based multilevel contrast stretching method to enhance image structure. This method directly operates on the objects that cannot cause the problems of ringing, blocking or false contouring artifacts. Lee et al. [1] used a space-variant luminance map (SVLM) for local brightness to enhance color images. The SVLM method is combined with 2D gamma correction to increase the dark intensity region and decrease the bright intensity region.

All the above-mentioned methods have the disadvantage that the time complexity is very high. Furthermore, using these methods requires that human beings determine many parameters for enhancement in advance of applying the method.

Herein, an adaptive local power-law transformation (ALPLT) will be proposed to enhance color images and avoid these disadvantages. The proposed method is simple, fast, requires only one window size parameter, and is enhanced automatically. Data are presented to analyze the performance of the window size parameter. In particular, the parameters in the local power-law transformation function are chosen automatically. In other words, it is nearly parameter-free, and it doesnt require intervention by a human operator.

The steps of the proposed system include: First, a color image is initially transformed into the HSV color space. Then, an adaptive local power-law transformation
(ALPLT) is applied to enhance the original color image. Finally, the backward color transformation is applied to produce the enhancement result.

\section{Adaptive Local Power-Law Transformation}

\subsection{Color Transformation}

There are many color models $[2,15]$. In this paper, the input color image with RGB color model is adopted. Next, the RGB color model is transformed to HSV color model. The reasons of using HSV color model are, in summary: First, the value V (luminance) and color information (Hue and Saturation) are decoupled. Second, the HSV color model describes perceptual color relationships more accurately than RGB color model. Third, transforming RGB color model to HSV color model is easy. Fourth, transforming HSV color model to RGB color model is easy too. (The transformation from RGB to HSV and the transformation from HSV to RGB can be found in [15]).

\subsection{Power-Law Transformation}

Power-law transformation is useful for general-purpose contrast enhancement. The power-law transformation [2] has the basic form

$$
O=c I^{\gamma}
$$

where $c$ and $\gamma$ are positive constants. Plots of $O$ versus $I$ for various values of $\gamma$ are shown in Fig. 1. A family of possible transformation curves are obtained simply by varying $\gamma$. The curves generated with values of $\gamma>1$ have exactly the opposite effect to those generated with values of $\gamma<1$. Further, when $c=\gamma=1$, (1) reduces to the identity transformation.

The ability to automatically determine $\gamma$ is very important for determining the best enhancement. How the method proposed here makes this automatic determination is described in Section 2.3 below.

\subsection{Adaptive Local Power-Law Transformation}

Global contrast enhancement cannot enhance both lowlight and highlight image with satisfactory results. However, local contrast enhancement uses the neighboring pixels to enhance both shadow and highlight image with satisfactory results. The proposed adaptive local power-law transformation (ALPLT) enhances each pixel by shifting a rectangular window across the image. The power-law transformation, in (1), is used in the rectangular window to enhance the treated image. 


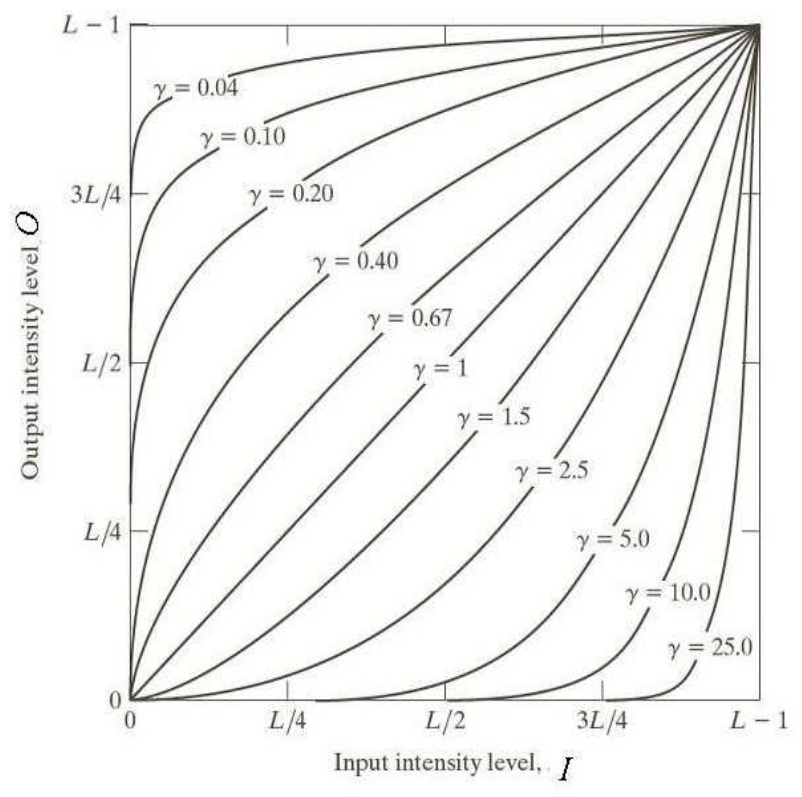

Fig. 1 Plots of (1) for various values of $\gamma(c=1$ in all cases). Modified from [2].

The proposed ALPLT method used is modified from $[1,13,16]$ and can be written as following equation:

$$
E(x, y)=255\left(\frac{f(x, y)}{255}\right)^{y}, \gamma=\left(\frac{1}{\alpha}\right)^{\left(\frac{128-\mu(x, y)}{128}\right)}
$$

where $E(x, y)$ represents the enhancement luminance value, $f(x, y)$ represents the original luminance value, $\gamma$ represents the exponential value, $\alpha$ is 2.2 which is commonly used in gamma correction for most computer systems and most standard displays, and

$$
\mu(x, y)=\frac{1}{s^{2}}\left[\sum_{j=y-\frac{s}{2}}^{y+\frac{s}{2}}\left(\sum_{i=x-\frac{s}{2}}^{x+\frac{s}{2}} f(i, j)\right)\right]
$$

where $\mu(x, y), x, y$, and $s$ are the local mean value in a local window, the index of the image width, the index of the image height, and the side of the local window, respectively.

From (2), a dark region in the image will have the exponential value $\gamma<1$ and a bright region in the image will have the exponential value $\gamma>1$. For example, for a dark region with the local mean $\mu(x, y)$ is 64 , the exponential value $\gamma \approx 0.6742$. This exponential value can expand the dark region to be the bright one. A bright region example is that if the local mean $\mu(x, y)$ is 192 , the exponential value $\gamma \approx 1.4832$. This exponential value can shrink the bright region to be the dark one.

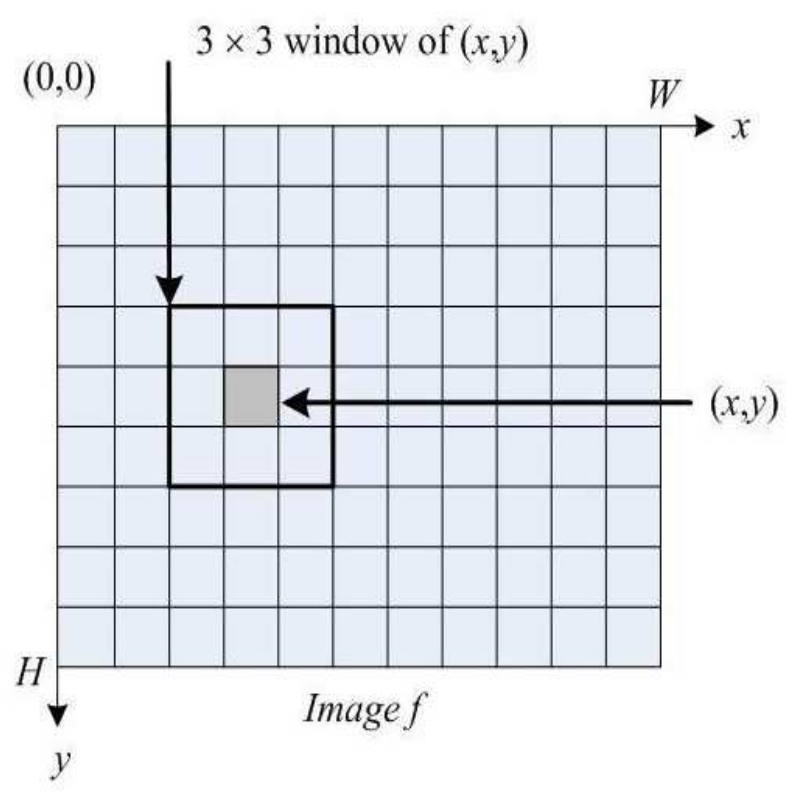

Fig. 2 A $3 \times 3$ window about a point $(x, y)$ in an image. The window is moved from pixel to pixel in the image to generate an enhanced image.

The conventional approach for computing the local mean has been shown in (3). Fig. 2 shows the basic implementation of (3) on a single image. The point $(x, y)$ shown is an arbitrary location in the image, and the small window shown containing the point is a neighborhood of $(x, y)$. The process that Fig. 2 illustrates consists of moving the origin of the window from pixel to pixel and applying the operation in (3) to the pixels in the window to yield the enhanced result at that location. Thus, for any specific location $(x, y)$, the value of the enhanced image $E$ at those coordinates is equal to the result of applying (3) to the neighborhood with origin at $(x, y)$ in $f$. For example, assume the window is a square of size $3 \times 3$ as in Fig. 2, and that (3) is defined so as to compute the average intensity of the window. The enhanced result, $E(x, y)$, at that location is computed as the sum of $f(x, y)$ and its 8 -neighbors, divided by 9 . The origin of the window is then moved to the next location and the procedure is repeated to generate the next value of the enhanced image $E$. Typically, the process starts at the top left of the input image and proceeds pixel by pixel in a horizontal scan, one row at a time.

However, this approach is a brute-force approach. Every pixel values in the local window must be summed to obtain the total pixel value. The total pixel value in the local window is then divided by the local window size $s \times s$ to obtain the local mean. In the general case, this algorithms per-pixel complexity is $O\left(s^{2}\right)$ in the local window. For one luminance image $f$ with width, $W$, and 

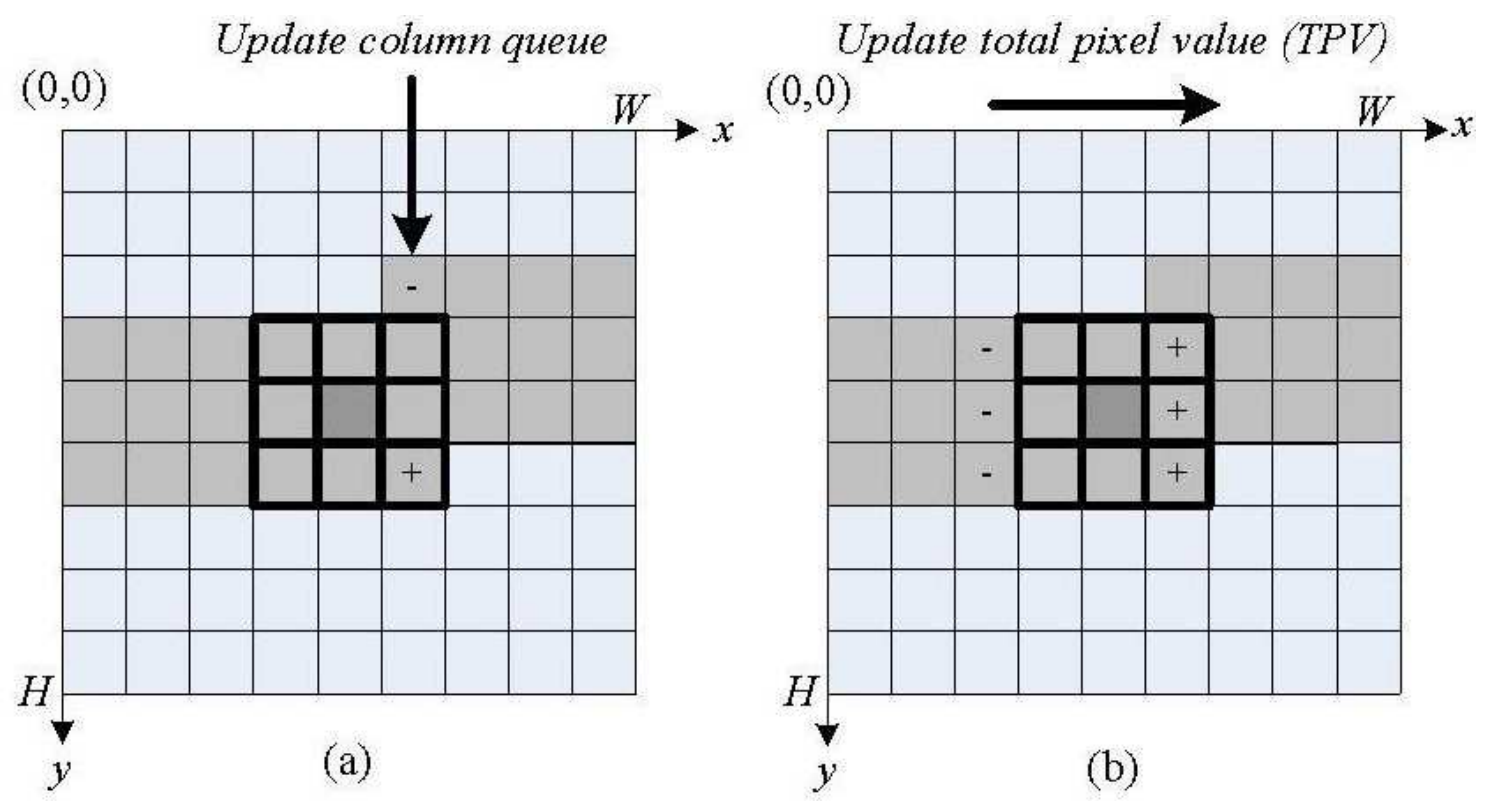

Fig. 3 The column queues. (a) The right column queue is updated by removing the top pixel value and adding the bottom one. (b) The total pixel value is updated by subtracting the leftmost column queue and adding the newly modified right column queue. Modified from $[17,18]$.

height, $H$, the total time complexity is $O\left(W H s^{2}\right)$. This approach is too time-consuming for most consumer products.

To overcome this problem, an efficient method is proposed to compute the local mean. It will be described in the balance of this paper.

\subsection{Computing Local Mean in Constant Time}

As explained above, local methods yield better results than global but are inefficient. Therefore, a computing local mean in constant time algorithm is proposed to reduce the time complexity for the proposed adaptive local power-law transformation.

Equation (3) can be divided into two parts and can be rewritten as follows:

$$
T P V=\left[\sum_{j=y-\frac{s}{2}}^{y+\frac{s}{2}}\left(\sum_{i=x-\frac{s}{2}}^{x+\frac{s}{2}} f(i, j)\right)\right]
$$

and

$$
\mu(x, y)=\frac{1}{s^{2}} T P V
$$

where TPV represents the total pixel value. This value is obtained by summing the pixel value in the local window. From (4), every pixel in the local window must be summed to find the total pixel value (TPV). That is, when no information is retained between rows, each pixel will need to be added many times over the whole image, which causes the $O\left(s^{2}\right)$ complexity in the local window. However if, as proposed herein, the information is retained between rows, the complexity for each pixel is at most a constant amount of time, measured by $O(1)$. The proposed means of computing the local mean with a constant time algorithm is based on $[17,18]$ and will be described as follows.

The proposed algorithm maintains one queue for each column in the image. These column queues are preserved across rows for the entirety of the process. Each column queue retains $s$ adjacent pixels. Fig. 3 illustrates the basic idea behind the construction of the column queues. These column queues are used to help computing the local mean with constant time. The total pixel value (TPV) is computed by summing $s$ adjacent column queues.

Consider the case of moving to the right from one pixel to the next. The column queue in the right is updated by removing its top pixel value and adding one new pixel below it (Fig. 3(a)). This action is caused by the column queue moving down one row. The complexity of this action is only $O(1)$ since one addition and one subtraction, independent of the window size, are necessary.

The total pixel value (TPV) is updated by similarly subtracting the leftmost column queue and adding the 


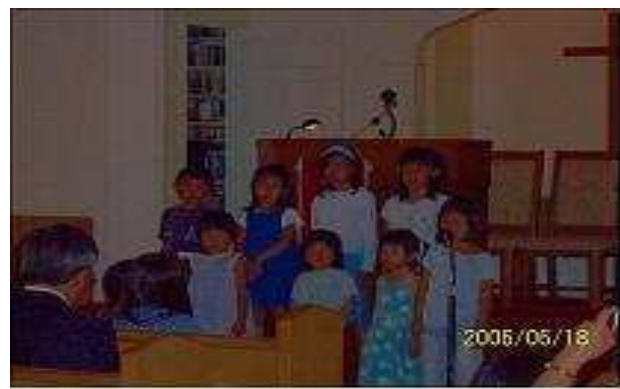

(b)

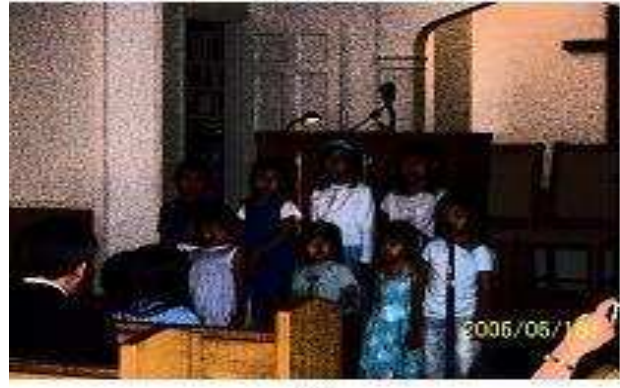

(d)

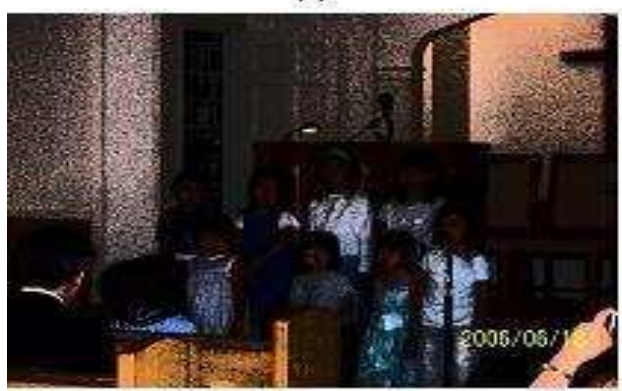

(f)

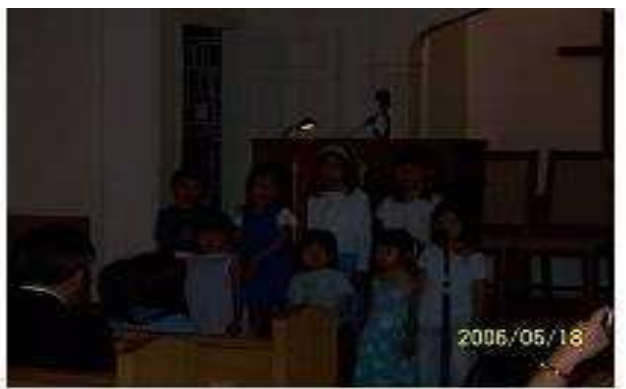

(a)

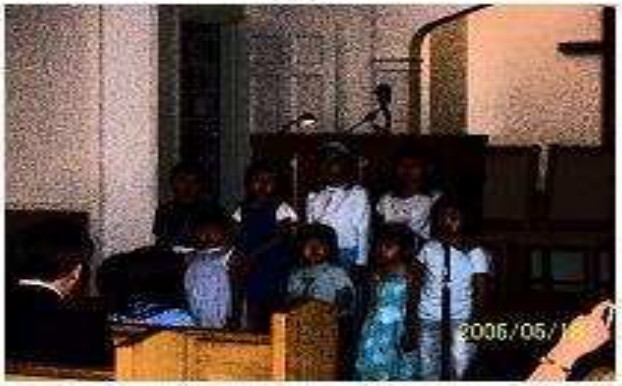

(c)

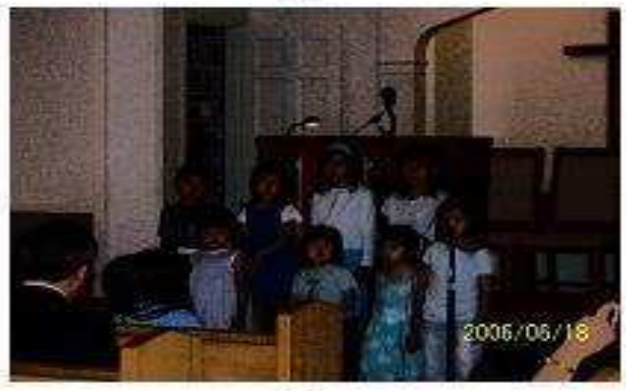

(e)

Fig. 4 Comparisons with conventional global enhancement methods. (a) Original image, (b) Proposed image $(s=15)$, (c) BBHEed image, (d) DSIHEed image, (e) MWCVMHEed image $(k=5)$, and (f) MMLSEMHEed image $(k=6)$.

newly modified right column queue (Fig. 3(b)) to update the new total pixel value (TPV) in the new local window when the current pixel is moving to right one pixel. The complexity of this action is only $O(1)$ too, since s additions and s subtractions are used.

Equation (5) is the last action for computing the local mean. The complexity of this action is also $O(1)$, since only one division or multiplication is involved. Furthermore, if the local window size $s \times s$ is set as power of 2 , the one division or multiplication can be replaced by the bitwise shift operator.

For the initialization problem, each column queue is initialed from the first $\mathrm{s}$ rows pixels. The total pixel value (TPV) is computed from the first $s$ column queues. The complexity of this initialization is $O(s)$. Of course, there is overhead when moving from one row to next row which requires determining a new $O(s)$. However, the initialization complexity is insignificant for large images because the amortized cost per pixel is still $O(1)$ when the image size is proportional to the local window size.

\section{Experiments}

The proposed ALPLT algorithm was implemented as a C \# 2010 application on a CPU $2.2 \mathrm{GHz}$ and memory 1.96GB Notebook. Our experiments use various color images. These images are obtained from [19], the internet, and were captured by us. The analysis of the experimental results compared the enhancement result of the proposed method with the enhancement results obtained by conventional global and local methods. 


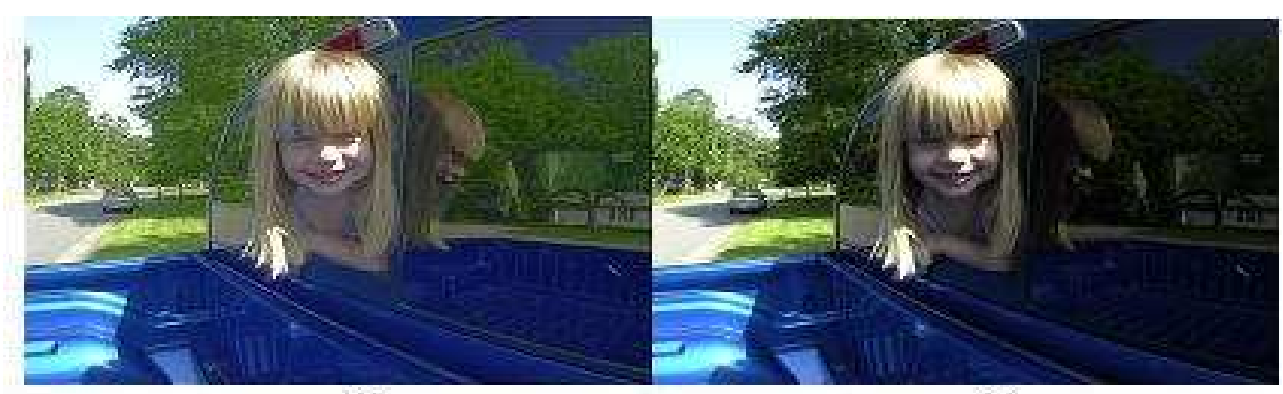

(b)

(a)

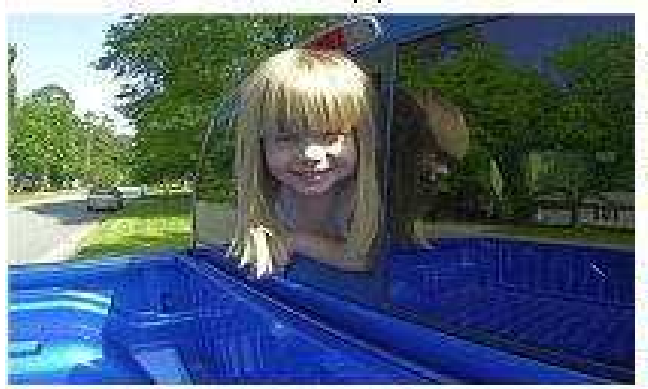

(d)

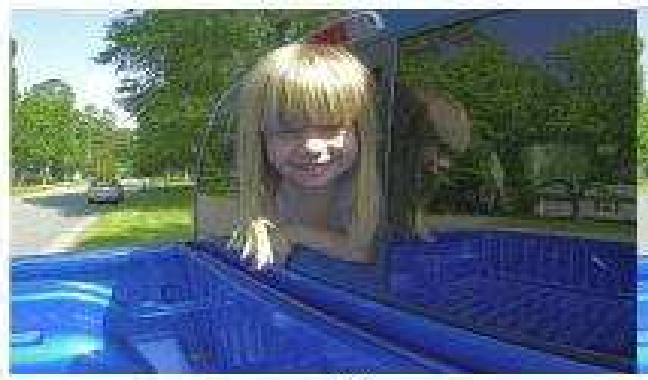

(f)

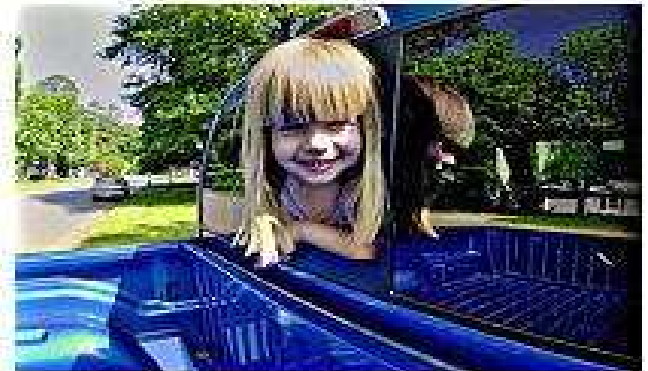

(c)

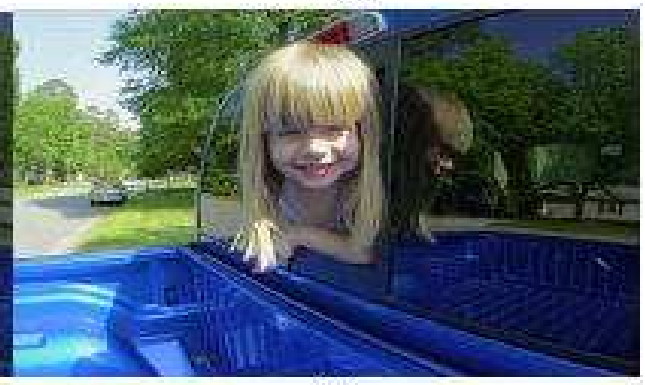

(e)

Fig. 5 Comparisons with conventional local enhancement methods. (a) Original image, (b) Proposed image $(s=15)$, (c) SVLMed image $(\gamma=0.5$ ), (d) MSRCRed image, (e) AINDANEed image, and (f) DROed image.

\subsection{Comparisons with Global Enhancement Methods}

The enhancement result of the proposed method is compared with the other enhancement results that were obtained by means preserving bi-histogram equalization (BBHE) [3], equal area dualistic sub-image histogram equalization (DSIHE) [4],MWCVMHE and MMLSEMHE global methods [5]. Figure 4 shows the enhancement results using a children's choir image. Figures 4(a)-4(f) are original image, Proposed image $(s=$ 15), BBHEed image, DSIHEed image, MWCVMHEed image $(k=5)$, and MMLSEMHEed image $(k=6)$ methods, respectively. The original image has the low contrast. The image size is $2048 \times 1536$. An observed looking at this image in its unenhanced form cannot see clearly how many children are singing in the choral. From this figure, it is evident that the enhancement results of the BBHE, DSIHE, MWCMHE, and MMLSEMHE methods do not show clearly the number of singers. On the other hand, using the proposed enhancement method yielded a better enhanced image and produced a more natural look than the comparison global enhancement methods.

\subsection{Comparisons with Local Enhancement Methods}

The enhancement result of the proposed method was also compared with the enhancement results by local enhancement methods, specifically the SVLM [1], MSRCR [9], AINDANE [12], and DRO [13] methods. Figure 5 shows the enhancement results for a non-uniform illumination image obtained from [19]. The image size is $2000 \times 1312$. Figures 5(a)-5(f) are original image, proposed image $(s=15)$, SVLMed image $(\gamma=$ $0.5)$, MSRCRed image, AINDANEed image, and DROed 
Table 1 Speed comparison for $2000 \times 1312$ test images

\begin{tabular}{|c|c|}
\hline Methods & Execution Time in Average \\
\hline Proposed $(\mathrm{s}=15)$ & 2.421 (second) \\
\hline AINDANE & 3.554 (second) \\
\hline SVLM $(=0.5)$ & 12.281 (second) \\
\hline MSRCR & 16.307 (second) \\
\hline DRO & 49.981 (second) \\
\hline
\end{tabular}

image methods, respectively.

Although the SVLM method gives the fine details, well-balanced contrast and luminance, and appropriate color saturation, the cost is very high. This method used the Gaussian mask to extract the local characteristic of the input intensity and used the bi-cubic interpolation to restore the down-scaled convolution images. Moreover, the hair in the mirror is not clearly shown. The MSRCR method gives the strong color enhancement, but noise, incorrect light, and the halo artifact are evident. The AINDANE method uses the separated enhancement methods to show the detail of the image. However, the colors in the tree and the sky are changed into lighter colors. The DRO method gives a blur effect and the details are lost. The enhancement result of the proposed method has more natural look than other comparison methods. Furthermore, the time complexity is very low.

\subsection{Speed Comparison for Local Enhancement Methods}

To compare the performance of the method in terms of execution time for enhancement of the tested images of size $2000 \times 1312$, obtained from [19]. Table 1 shows the execution time for each local method. From this table, it is evident that the proposed method takes the least execution time of all the compared local enhancement methods.

\subsection{Performance for Computing Local Mean}

To demonstrate the performance of the proposed algorithm to compute the local mean, the tested images are adopted from [19]. The performances using and not using the proposed speedup algorithm are shown. The window size for both use and non-use of the proposed speedup algorithm is 33 . Table 2 shows the results for computational time. From this table, the proposed speedup algorithm is shown to be 5.353 faster than the non-speedup method. Thus, the proposed speedup algorithm is very efficient.
Table 2 Computational times for use and not use the proposed speedup algorithm

\begin{tabular}{|c|c|}
\hline Not use speedup & 9.946 (second) \\
\hline Use speedup & 1.858 (second) \\
\hline
\end{tabular}

\section{Conclusions}

This study has presented an adaptive local power-law transformation method to enhancement of color images. The proposed method also employed a very efficient method to compute the local mean. The performance analysis indicated that the proposed method is very efficient and effective in comparison to conventional global and local enhancement methods including the global methods include BBHE, DSIHE, MWCVMHE, and MMLSEMHE methods and local methods include SVLM, MSRCR, AINDANE, and DRO methods.

\section{Acknowledgements}

The author would like express his gratitude to Dr. Walter Slocombe, JD. and Dr. Jeffrey Lee, for their assistance in English writing. This work was supported by the National Science Council, R.O.C., under Grants NSC 100-2221-E-133-004-. The author thanks the reviewers for their comments.

\section{References}

[1] S. M. Lee, H. M. Kwon, H. Y. Han, G. D. Lee, and B. S. Kang, A space-variant luminance map based color image enhancement. IEEE Trans. Consumer Electronics, 56, 26362643 (2010).

[2] R. C. Gonzalez and R. E. Woods, Digital Image Processing, 3rd Ed., Prentice Hall, (2008).

[3] Y. T. Kim, Contrast enhancement using brightness preserving bi-histogram equalization. IEEE Trans. Consumer Electronics, 43, 1-8 (1997).

[4] Y. Wang, Q. Chen, and B. Zhang, Image enhancement based on equal area dualistic sub-image histogram equalization method. IEEE Trans. Consumer Electronics, 45, 68-75 (1999).

[5] D. Menotti, L. Najman, J. Facon, and A. de A. Araujo, Multi-histogram equalization methods for contrast enhancement and brightness preserving. IEEE Trans. Consumer Electronics, 53, 1186-1194 (2007).

[6] B. N. Chatterji and N. R Murthy, Adaptive contrast enhancement for color images. In Proc. of 1997 International Conference on Information, Communications and Signal Processing, 3, 1537-1541 (1997).

[7] L. Meylan and S. Ssstrunk, Color image enhancement using a retinex-based adaptive filter. In Proc. IS\&T Second European Conference on Color in Graphics, Image, and Vision. April, 2, 359-363 (2004). 
[8] C. Munteanu and A. Rosa, Color image enhancement using evolutionary principles and the retinex theory of color constancy. Neural Networks for Signal Processing XI, 2001. In Proc. of the 2001 IEEE Signal Processing Society Workshop, 393-402 (2001).

[9] D. Jobson, Z. Rahman, and G. Woodell, A multiscale retinex for bridging the gap between color images and the human observation of scenes. IEEE Trans. Image Processing, 6, 965-976 (1997).

[10] A. Choudhury and G. Medioni, Perceptually motivated automatic color contrast enhancement based on color constancy estimation. EURASIP Journal on Image and Video Processing, 2010, 1-22 (2010).

[11] R. Kimmel, M. Elad, D. Shaked, R. Keshet, and I. Sobel, A variational framework for retinex. International Journal of Computer Vision, 52, 7-23 (2003).

[12] L. Tao and V. K. Asari, Adaptive and integrated neighborhood-dependent approach for nonlinear enhancement for color images. Journal of Electronics Imaging, 14, 1-14 (2005).

[13] A. Capra, A. Castorina and S. Corchs, Dynamic range optimization by local contrast correction and histogram image analysis. ICCE, 2006, 309-310 (2006).

[14] B. L. Xu, Y. Q. Zhuang, H. L. Tang, and L. Zhang, Objectbased multilevel contrast stretching method for image enhancement. IEEE Trans. Consumer Electronics, 56, 17461754 (2010).

[15] C. M. Tsai and Z. M. Yeh, Contrast enhancement by automatic and parameter-free piecewise linear transformation for color images. IEEE Trans. Consumer Electronics, 54, 213-219 (2008).

[16] N. Moronry, Local color correction using non-linear masking. IS\&T/SID 8th color imaging conference. Scottsdale, 108-111 (2000).

[17] S. Perreault and P. Hebert, Median filtering in constant time. IEEE Trans. Image Processing. 16, 2389-2394 (2007).

[18] M. Sizintsev, K. G. Derpanis, and A. Hogue, Histogrambased search: A comparative study, 2008, 23-28 (2008).

[19] Retinex image processing, NASA website: http://dragon.larc.nasa.gov/retinex/pao/news/.

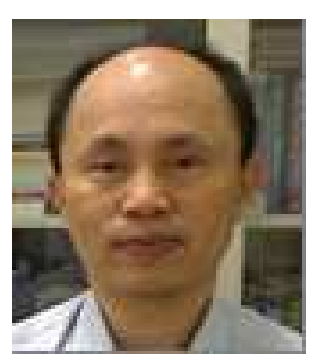

\section{Chun-Ming}

Tsai received the M.S. and the Ph. D. degrees in Computer Science and Information Engineering from National Chiao Tung University, Hsinchu, Taiwan in 1996 and 2002, respectively. He was a visiting scholar in Department of Computer Science, University of California Santa Barbara, California, USA, 2005, 8 2006, 7. He is currently a Professor in Department of Computer Science, Taipei Municipal University of Education. His research interests include Video and Image Processing, Machine Learning, Medical Image
Processing, Document Image Analysis, and Brain Computer Interface. 